04

\title{
Влияние коронного разряда на оптические свойства тонкопленочных структур $\mathrm{Cu}-\mathrm{As}_{2} \mathrm{Se}_{3}$
}

\author{
(C) А.М. Настас ${ }^{1}$, М.С. Иову ${ }^{1}$, А.Л. Толстик ${ }^{2}$ \\ ${ }^{1}$ Институт прикладной физики, \\ MD-2028 Кишинев, Молдова, \\ ${ }^{2}$ Белорусский государственный университет, \\ 220030 Минск, Беларусь \\ e-mail: nastas_am@rambler.ru
}

Поступила в редакцию 17.102019 г.

В окончательной редакции 17.10.2019 г.

Принята к публикации 28.10.2019 г.

Изучено влияние поля коронного разряда на голографическую запись дифракционных решеток в тонкопленочной структуре $\mathrm{Cu}-\mathrm{As}_{2} \mathrm{Se}_{3}$, полученной методом термического осаждения в вакууме на стеклянных подложках. Установлено, что приложение отрицательного коронного разряда во время записи приводит к увеличению голографической чувствительности структуры $\mathrm{Cu}-\mathrm{As}_{2} \mathrm{Se}_{3}$ и дифракционной эффективности решеток. Облучение актиничным светом приводило к потемнению структуры $\mathrm{Cu}-\mathrm{As}_{2} \mathrm{Se}_{3}$ в спектральной области сильного поглощения и просветлению в области слабого поглощения. Приложение положительного коронного разряда приводило к усилению фотопросветления, а отрицательного коронного разряда — к его ослаблению. Полученные экспериментальные результаты качественно объясняются в предположении диффузии ионов $\mathrm{Cu}^{+}$в пленку $\mathrm{As}_{2} \mathrm{Se}_{3}$.

Ключевые слова: халькогенидные пленки, тонкопленочная структура, дифракционная эффективность, спектр пропускания, коронный разряд.

DOI: $10.21883 /$ OS.2020.02.48967.283-19

\section{Введение}

Халькогенидным стеклообразным полупроводникам (ХСП), несмотря на их давнее открытие (1956 г.), уделяется повышенное внимание и сегодня. Большое внимание уделяется прежде всего электрическим и оптическим свойствам тонких некристаллических пленок ХСП, например, в работах [1-3].

Основными областями развития оптических технологий являются разработка новых материалов, в том числе и регистрирующих сред, а также разработка новых способов оптической записи. В качестве регистрирующих структур для оптической голографической записи перспективным является использование тонких некристаллических пленок ХСП. Наиболее востребованы способы оптической записи в тонких пленках ХСП за счет фотоструктурных превращений (ФСП) и фотодиффузии металла (ФДМ) в структуре металл-ХСП [4]. Следует отметить, что эти классические способы оптической записи были модернизированы [5]. В работе [5] предложено проводить экспонирование с одновременной зарядкой структуры металл-ХСП в поле коронного разряда. Этот способ записи приводит к увеличению в несколько раз чувствительности, дифракционной эффективности и глубины рельефа, полученного после химического травления [6] как за счет ФСП [7-9], так и при ФДМ [10,11]. Следует отметить, что при использовании тонких слоев ХСП влияние коронного разряда проявляется еще ярче. Так, максимально достижимое значение дифракционной эффективности при использовании коронного разряда выше, чем при обычной записи (без коронного разряда) за счет ФСП примерно в 30 и 10 раз для толщины пленки ХСП соответственно 29 и $56 \mathrm{~nm}$ [12].

Целью данной работы являлось исследование влияния коронного разряда на фотоиндуцированное изменение спектров пропускания структур $\mathrm{Cu}-\mathrm{As}_{2} \mathrm{Se}_{3}$ и на запись в них голографических дифракционных решеток.

\section{Методика получения и исследования образцов}

Тонкопленочные структуры $\mathrm{Cu}-\mathrm{As}_{2} \mathrm{Se}_{3}$ были получены методом последовательного термического испарения в вакууме (давление $3.7 \cdot 10^{-3} \mathrm{~Pa}$ ) слоев $\mathrm{Cu}$ и ХСП на стеклянные подложки при скорости осаждения ХСП около $5 \mathrm{~nm} / \mathrm{s}$. Толщина слоя ХСП в разных случаях была равна 0.27 и $0.11 \mu \mathrm{m}$. Толщина металлического слоя $\mathrm{Cu}$ была равна примерно $40 \mathrm{~nm}$. Свежеприготовленные структуры $\mathrm{Cu}-\mathrm{As}_{2} \mathrm{Se}_{3}$ были использованы для изучения спектров пропускания, а также для голографической записи дифракционных решеток. Следует отметить, что слой меди при записи в поле коронного разряда служил одним из электродов, а пропускание медной пленки на длине волны $0.63 \mu \mathrm{m}$ составляло около $30 \%$.

Для изучения спектров пропускания структуры $\mathrm{Cu}$ $\mathrm{As}_{2} \mathrm{Se}_{3}$ проводилось ее облучение со стороны ХСП Не$\mathrm{Ne}$-лазером (длина волны $\lambda=0.6328 \mu \mathrm{m}$, экспозиция 
$\left.H=0.5 \mathrm{~J} / \mathrm{cm}^{2}\right)$ как при приложении поля коронного разряда $( \pm 7 \mathrm{kV})$, так и без него. Измерения спектров пропускания производились с помощью компьютеризованного спектрофотометра SPEECORD UV-VIS.

Коронирующее устройство состояло из высоковольтного блока и коронатора. Коронирующим электродом была тонкая $(60 \mu \mathrm{m})$ вольфрамовая нить, на которую подавался потенциал $+7 \mathrm{kV}$ или $-7 \mathrm{kV}$. Расстояние между нитью и поверхностью образцов составляло $17 \mathrm{~mm}$.

Голографическая запись решеток осуществлялась как в поле коронного разряда, так и без него. Запись голографических дифракционных решеток с периодом $2 \mu \mathrm{m}$ осуществлялась по стандартной внеосевой схеме, в сходящихся пучках Не-Ne-лазера $(\lambda=0.6328 \mu \mathrm{m})$. Измерение дифракционной эффективности $\eta$ производилось в первом порядке дифракции в прошедшем свете также с $\lambda=0.6328 \mu \mathrm{m}$ при его нормальном падении на поверхность слоя ХСП. Значение дифракционной эффективности определялось как отношение интенсивности света в первом дифракционном максимуме к интенсивности света, прошедшего через неэкспонированный участок используемого образца.

\section{Экспериментальные результаты и их обсуждение}

На рис. 1 (аналогичный рисунок приведен в работе [11]) показаны зависимости дифракционной эффективности от экспозиции для голографических дифракционных решеток с пространственной частотой $500 \mathrm{~mm}^{-1}$, сформированных как при обычной записи (кривые 2, 5), так и при приложении поля коронного разряда во время записи (кривые 1,3,4). Как видно из рисунка, использование отрицательного коронного разряда приводило к увеличению как чувствительности структуры $\mathrm{Cu}-\mathrm{As}_{2} \mathrm{Se}_{3}$, так и дифракционной эффективности записанных решеток (сравни попарно кривые 1,2 и 4,5). Напротив, использование положительного коронного разряда приводило к уменьшению как чувствительности, так и дифракционной эффективности решеток (сравни кривые 2 и 3 ).

Полученные результаты подобно работе [11] легко могут быть качественно объяснены в рамках существующей фотоэлектрической модели $[4,13]$. В этой модели предполагается, что скорость процесса фотодиффузии ограничивается скоростью проникновения металла из легированной области ХСП в нелегированную. При освещении светом на границе легированного и нелегированного слоев ХСП происходит разделение электронов и дырок таким образом, что они создают тянущее электрическое поле для ионов металла [4]. При приложении к структуре $\mathrm{Cu}-\mathrm{As}_{2} \mathrm{Se}_{3}$-поля коронного разряда суммарное тянущее электрическое поле, в котором перемещаются положительные ионы металла из фотолегированного слоя в нелегированный слой $[4,13]$, будет увеличиваться при отрицательном коронном разряде и уменьшаться при положительном коронном разряде.



Рис. 1. Зависимости дифракционной эффективности от экспозиции для голографических дифракционных решеток, сформированных в структуре $\mathrm{Cu}-\mathrm{As}_{2} \mathrm{Se}_{3}$ при различных условиях записи: без коронного разряда (2 и 5), в поле отрицательного коронного разряда при $U=-7 \mathrm{kV}$ ( 1 и 4), в поле положительного коронного разряда $U=+7 \mathrm{kV}(3)$. Толщина слоя ХСП составляет $0.27 \mu \mathrm{m}$ (кривые 1,2 и 3) и $0.11 \mu \mathrm{m}$ (4 и 5 ).

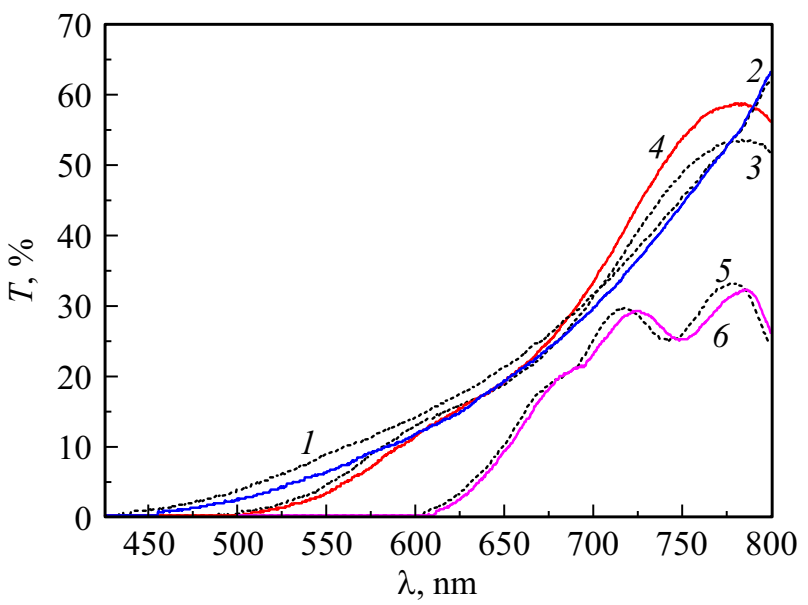

Pис. 2. Спектры пропускания структур $\mathrm{Cu}-\mathrm{As}_{2} \mathrm{Se}_{3}(1-4)$ и $\mathrm{Al}-$ $\mathrm{As}_{2} \mathrm{Se}_{3}$ (5 и 6), полученные до $(1,3,5)$ и после $(2,4,6)$ их облучения. Толщина слоя $\mathrm{As}_{2} \mathrm{Se}_{3}$ составляет $0.11 \mu \mathrm{m}$ (1 и 2), $0.27 \mu \mathrm{m}(3$ и 4$)$ и $1 \mu \mathrm{m}(5$ и 6$)$.

На рис. 2 представлены спектры пропускания свежеприготовленных структур металл- $\mathrm{As}_{2} \mathrm{Se}_{3}$ до (кривые 1,3 и 5) и после их облучения (кривые 2,4 и 6) без приложения поля коронного разряда. Кривые 1 и 2 соответствуют толщине пленки $\mathrm{As}_{2} \mathrm{Se}_{3}$, равной $0.11 \mu \mathrm{m}$, кривые 3 и 4 - толщине пленки $\mathrm{As}_{2} \mathrm{Se}_{3}$, равной $0.27 \mu \mathrm{m}$, а кривые 5 и 6 - толщине $\mathrm{As}_{2} \mathrm{Se}_{3} 1 \mu \mathrm{m}$. Для сравнения на рис. 2 приведены также спектральные зависимости пропускания структуры $\mathrm{Al}-\mathrm{As}_{2} \mathrm{Se}_{3}$, измеренные до (кривая 5) и после облучения (кривая 6). В этом случае толщина слоя $\mathrm{As}_{2} \mathrm{Se}_{3}$ составляла $1 \mu \mathrm{m}$, а толщина слоя $\mathrm{Al}$ в несколько десятков нанометров обладала коэффициентом пропускания на длине волны $\lambda=0.63 \mu \mathrm{m}$, равным 


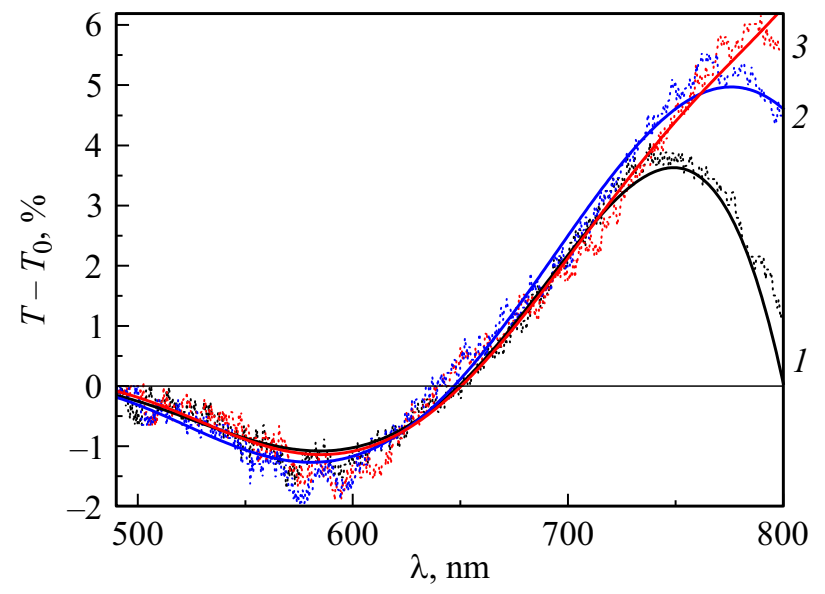

Pис. 3. Спектральные зависимости фотоиндуцированного изменения оптического пропускания структуры $\mathrm{Cu}-\mathrm{As}_{2} \mathrm{Se}_{3}$ с толщиной слоя ХСП, равной $0.27 \mu \mathrm{m}$, при оптическом облучении в поле коронного разряда при $U=-7 \mathrm{kV}$ (1), $0 \mathrm{kV}$ (2) и $+7 \mathrm{kV}(3)$.



Рис. 4. Спектральные зависимости фотоиндуцированного изменения оптического пропускания структуры $\mathrm{Cu}-\mathrm{As}_{2} \mathrm{Se}_{3}$ с толщиной слоя ХСП, равной $0.11 \mu \mathrm{m}$, при оптическом облучении в поле коронного разряда при $U=-7 \mathrm{kV}$ (1) и $0 \mathrm{kV}(2)$.

примерно 50\%. Известно, что в структуре $\mathrm{Cu}-\mathrm{As}_{2} \mathrm{Se}_{3}$ при облучении светом наблюдается фотодиффузия ионов $\mathrm{Cu}$ в пленку ХСП, в то время как в структуре $\mathrm{Al}-\mathrm{As}_{2} \mathrm{Se}_{3}$ фотодиффузия ионов $\mathrm{Al}$ в пленку ХСП не происходит. При облучении структуры $\mathrm{Al}-\mathrm{As}_{2} \mathrm{Se}_{3}$ актиничным светом происходят только фотоструктурные превращения, сопровождающиеся фотопотемнением, что видно из рис. 2 (сравни кривые 5 и 6). Как видно из рис. 2, в отличие от структуры $\mathrm{Al}-\mathrm{As}_{2} \mathrm{Se}_{3}$ для структуры $\mathrm{Cu}-\mathrm{As}_{2} \mathrm{Se}_{3}$ в области слабого поглощения наблюдается фотопросветление, обусловленное уменьшением толщины медной пленки. Максимумы на кривых 3 и 4 (толщина пленки $\left.\mathrm{As}_{2} \mathrm{Se}_{3} 0.27 \mu \mathrm{m}\right)$ обусловлены интерференцией света в тонких пленках.

Влияние условий облучения на спектральное распределение фотоиндуцированного изменения спектров пропускания $\Delta T(\lambda)=T(\lambda)-T_{0}(\lambda)$ структуры $\mathrm{Cu}-\mathrm{As}_{2} \mathrm{Se}_{3}$ представлено семейством кривых на рис. 3 и 4 для толщины слоя $\mathrm{As}_{2} \mathrm{Se}_{3}$, равной 0.27 и $0.11 \mu \mathrm{m}$ соответственно. Здесь $T_{0}(\lambda)$ и $T(\lambda)$ обозначают пропускание исследуемой структуры до и после облучения. На каждом из двух рисунков кривая 2 соответствует облучению без приложения поля коронного разряда (обычное облучение), а кривая 1 - облучению при приложенном отрицательном поле коронного разряда $(V=-7 \mathrm{kV})$. Кривая 3 на рис. 3 соответствует облучению структуры в поле положительного коронного разряда $(+7 \mathrm{kV})$.

Спектральная область, в которой $T(\lambda)<T_{0}(\lambda)$, соответствует фотопотемнению структуры $\mathrm{Cu}-\mathrm{As}_{2} \mathrm{Se}_{3}$, индуцированному облучением, а спектральная область, в которой $T(\lambda)>T_{0}(\lambda)$, - фотопросветлению. В коротковолновой области спектра, соответствующей сильному поглощению света, кривые $1-3$ на рис. 3 практически совпадают. Это говорит о том, что приложение поля коронного разряда не оказывает влияние на фотопотемнение структуры $\mathrm{Cu}-\mathrm{As}_{2} \mathrm{Se}_{3}$, несмотря на то, что влияет на процесс фотодиффузии ионов меди в пленку $\mathrm{As}_{2} \mathrm{Se}_{3}$. В то же время приложение поля коронного разряда заметно влияло на фотопросветление структуры $\mathrm{Cu}$ $\mathrm{As}_{2} \mathrm{Se}_{3}$, наблюдаемое в длинноволновой спектральной области, соответствующей слабому поглощению света. Положительное поле коронного разряда усиливало фотопросветление (сравни кривые 3 и 2 на рис. 3), а отрицательное - ослабляло его (сравни кривые 1 и 2 на рис. 3 ).

Ослабление фотопросветления структуры $\mathrm{Cu}-\mathrm{As}_{2} \mathrm{Se}_{3}$ при ее облучении в поле отрицательного коронного разряда наблюдалось также и в случае более тонкого слоя $\mathrm{As}_{2} \mathrm{Se}_{3}$ (рис. 4). Отличие структуры $\mathrm{Cu}-\mathrm{As}_{2} \mathrm{Se}_{3}$ с толщиной пленки ХСП, равной $0.11 \mu \mathrm{m}$ (рис. 4), состоит в том, что при ее облучении в поле отрицательного коронного разряда положение минимума (наибольшее фотопотемнение) заметно сдвигается в длинноволновую область по сравнению со случаем облучения без приложения поля коронного разряда (сравни кривые 1 и 2 на рис. 4). При этом приложение поля отрицательного коронного разряда приводит к усилению фотопотемнения структуры $\mathrm{Cu}-\mathrm{As}_{2} \mathrm{Se}_{3}$.

Полученные результаты можно качественно объяснить, рассматривая структуру $\mathrm{Cu}-\mathrm{As}_{2} \mathrm{Se}_{3}$ в виде последовательности нескольких слоев, вносящих вклад в суммарное поглощение света. При этом необлученная структура состоит из слоя металла и чистой (нелегированной) пленки $\mathrm{As}_{2} \mathrm{Se}_{3}$, а в облученной структуре образуется также легированная ионами $\mathrm{Cu}$ область слоя $\mathrm{As}_{2} \mathrm{Se}_{3}$, прилегающая к металлическому электроду. То есть изначально однородный по толщине слой $\mathrm{As}_{2} \mathrm{Se}_{3}$ в результате фотодиффузии ионов меди разделяется на два подслоя. Толщина и соответственно поглощение составляющих структуру слоев различным образом изменяются в результате фотоиндуцированной диффузии ионов $\mathrm{Cu}$ в пленку $\mathrm{As}_{2} \mathrm{Se}_{3}$. Именно это обусловливает влияние условий облучения структуры $\mathrm{Cu}-\mathrm{As}_{2} \mathrm{Se}_{3}$ на ход полученных спектральных зависимостей пропускания. 
В работе [14] показано, что легирование пленки ХСП ионами $\mathrm{Ag}$ приводит к ее потемнению в коротковолновой области спектра. Аналогично фотолегирование подслоя $\mathrm{As}_{2} \mathrm{Se}_{3}$ ионами Сu приводит к длинноволновому сдвигу края поглощения света в нем и соответственно в структуре $\mathrm{Cu}-\mathrm{As}_{2} \mathrm{Se}_{3}$ в целом. В то же время процесс фотодиффузии ионов $\mathrm{Cu}$ из подстилающего электрода приводит к уменьшению толщины последнего и соответственно к уменьшению поглощения света в нем и в структуре в целом. При увеличении длины волны поглощение света в легированном подслое уменьшается слабее, чем в нелегированном подслое $\mathrm{As}_{2} \mathrm{Se}_{3}$, и тем более слабее, чем в необлученном слое $\mathrm{As}_{2} \mathrm{Se}_{3}$. Этим можно объяснить начальный участок спада приведенных спектральных зависимостей фотоиндуцированного изменения пропускания $\Delta T(\lambda)$. Участок нарастания при больших длинах волн, по-видимому, обусловлен тем, что при дальнейшем ослаблении поглощения в легированном слое $\mathrm{As}_{2} \mathrm{Se}_{3}$ заметный вклад в фотоиндуцированное изменение пропускания структуры $\mathrm{Cu}-\mathrm{As}_{2} \mathrm{Se}_{3}$ начинает вносить уменьшение поглощения света в подстилающем металлическом слое вследствие уменьшения его толщины. Таким образом, совокупность фотоиндуцированного увеличения поглощения света в слое $\mathrm{As}_{2} \mathrm{Se}_{3}$ и уменьшения поглощения света в металлическом слое приводит к появлению минимума на спектральной зависимости фотоиндуцированного изменения пропускания $\Delta T(\lambda)$.

Ослабление поглощения света слоем $\mathrm{As}_{2} \mathrm{Se}_{3}$ (в том числе в легированном подслое) приводит к тому, что уменьшение поглощения света в пленке $\mathrm{Cu}$ становится преобладающим фактором, определяющим пропускание структуры $\mathrm{Cu}-\mathrm{As}_{2} \mathrm{Se}_{3}$ в длинноволновой спектральной области. Это обусловливает проявление эффекта фотопросветления структуры $\mathrm{Cu}-\mathrm{As}_{2} \mathrm{Se}_{3}$ в указанной спектральной области.

Поле коронного разряда оказывает влияние на процесс фотодиффузии ионов $\mathrm{Cu}$ в пленку $\mathrm{As}_{2} \mathrm{Se}_{3}$. Тянущее электрическое поле, созданное в результате зарядки тонкопленочной структуры $\mathrm{Cu}-\mathrm{As}_{2} \mathrm{Se}_{3}$ отрицательным коронным разрядом, во время облучения усиливает диффузию ионов $\mathrm{Cu}$, обладающих положительным зарядом, а поле при зарядке положительным коронным разрядом проявляется как тормозящее и ослабляет диффузию ионов $\mathrm{Cu}$. Поэтому по сравнению с обычным облучением при приложении отрицательного коронного разряда образуются легированный подслой $\mathrm{As}_{2} \mathrm{Se}_{3}$, обладающий более сильным поглощением света, и слой меди, обладающий более высоким пропусканием света. И наоборот, приложение положительного коронного разряда приводит к формированию легированного подслоя $\mathrm{As}_{2} \mathrm{Se}_{3}$ с уменьшенным поглощением света и слоя меди с уменьшенным пропусканием. Ослабление эффекта фотопросветления при приложении отрицательного коронного разряда по сравнению с обычным облучением (кривые 1 и 2 на рис. 3) можно объяснить тем, что увеличенное поглощение в легированном подслое $\mathrm{As}_{2} \mathrm{Se}_{3}$ оказывается сильнее, чем увеличенное пропускание в слое меди.
Аналогично усиление фотопросветления при приложении положительного коронного разряда (кривые 3 и 2 на рис. 3) объясняется тем, что в этом случае по сравнению с обычным облучением происходит преимущественно уменьшение поглощения в легированном подслое $\mathrm{As}_{2} \mathrm{Se}_{3}$.

При таком рассмотрении экспериментальных результатов можно сделать вывод, что влияние поля коронного разряда, приложенного во время облучения структуры $\mathrm{Cu}-\mathrm{As}_{2} \mathrm{Se}_{3}$, на ее оптические свойства проявляется преимущественно в изменении пропускания слоя $\mathrm{As}_{2} \mathrm{Se}_{3}$.

В коротковолновой спектральной области заметное поглощение света происходит и в нелегированном слое $\mathrm{As}_{2} \mathrm{Se}_{3}$. В этом случае влияние поля коронного разряда на суммарное пропускание легированного и нелегированного подслоев $\mathrm{As}_{2} \mathrm{Se}_{3}$ является более слабым, чем в длинноволновой спектральной области. Возможно, поэтому в эксперименте не наблюдалось влияние поля коронного разряда на фотопотемнение структуры $\mathrm{Cu}$ $\mathrm{As}_{2} \mathrm{Se}_{3}$ с более толстым слоем $\mathrm{As}_{2} \mathrm{Se}_{3}$ (рис. 3). Следует ожидать, что влияние поля коронного разряда на пропускание структуры $\mathrm{Cu}-\mathrm{As}_{2} \mathrm{Se}_{3}$ со слоем $\mathrm{As}_{2} \mathrm{Se}_{3}$ меньшей толщины будет заметнее. Это подтверждается результатами изучения спектров пропускания структуры $\mathrm{Cu}-\mathrm{As}_{2} \mathrm{Se}_{3}$ со слоем As2Se3 толщиной $0.11 \mu \mathrm{m}$ (рис. 4).

Следует отметить, что исследование спектров фотоиндуцированного изменения пропускания $\Delta T(\lambda)$ позволяет выяснить вклад в абсолютное значение дифракционной эффективности амплитудной составляющей амплитуднофазовых голографических дифракционных решеток. Как видно из рисунков, изменение дифракционной эффективности, измеренной в прошедшем свете $(0.63 \mu \mathrm{m})$, обусловленное применением коронного разряда, происходит только за счет фазовой модуляции светового потока.

\section{Выводы}

1. Использование отрицательного коронного разряда при записи в структурах $\mathrm{Cu}-\mathrm{As}_{2} \mathrm{Se}_{3}$ приводит к увеличению голографической чувствительности структуры и дифракционной эффективности записанных голографических дифракционных решеток по сравнению с решетками, записанными без коронного разряда. Приложение поля положительного коронного разряда приводит к противоположному эффекту.

2. Облучение тонкопленочной структуры $\mathrm{Cu}-\mathrm{As}_{2} \mathrm{Se}_{3}$ актиничным светом приводит к изменению ее спектра пропускания. В коротковолновой части видимого спектра, соответствующей сильному поглощению света, наблюдается фотопотемнение, а в длинноволновой части, для которой характерно слабое поглощение, наблюдается фотопросветление.

3. На кривых фотоиндуцированного изменения спектра пропускания $\Delta T(\lambda)$ наблюдается минимум, который обусловлен, по-видимому, противоположным влиянием 
на пропускание структуры $\mathrm{Cu}-\mathrm{As}_{2} \mathrm{Se}_{3}$ снижения пропускания слоя $\mathrm{As}_{2} \mathrm{Se}_{3}$, вызванного фотолегированием ионами меди, и повышения пропускания пленки меди за счет уменьшения ее толщины.

4. Использование во время облучения положительного коронного разряда приводит к усилению просветления в длинноволновой части видимого спектра по сравнению с обычным облучением, а применение отрицательного коронного разряда - к ослаблению.

5. Изменение дифракционной эффективности, измеренной в прошедшем свете $(\lambda=0.63 \mu \mathrm{m})$, обусловленное применением коронного разряда, происходит только за счет фазовой модуляции светового потока.

\section{Финансирование работы}

Работа выполнена в рамках международного двустороннего Молдо-Белорусского проекта ANCD $\quad 1980013.50 .07 .05 \mathrm{~A} / \mathrm{BL}, \quad$ Ф19МЛДГ-001 и институционального проекта CSSDT 15.817.02.03A.

\section{Конфликт интересов}

Авторы заявляют, что у них нет конфликта интересов.

\section{Список литературы}

[1] Boolchandani S., Srivastava S., Vijay Y.K. // J. Nanotechnology. 2018. V. 2018. Article ID 9380573, 9 pages. doi $10.1155 / 2018 / 9380573$

[2] Morris J.M., Mackenzie M.D., Petersen C.R. // Optical Materials Express. 2018. V. 8. N 4. doi 10.1364/OME.8.001001

[3] Kumar A., Shukla S., Shukla R.K. // Materials Focus. 2017. V. 6. N 4. P. 415(9). doi 10.1166/mat.2017.1425

[4] Венгер Е.Ф., Мельничук А.В., Стронский А.В. Фотостимулированые процессы в халькогенидных стеклообразных полупроводниках и их практическое применение. Киев: Академпериодика, 2007. С. 285.

[5] Nastas A.M., Andriesh A.M., Bivol V.V., Prisakar A.M., Tridukh G.M. Inventor's Certificate 3330 (MD), MD - BOPI nr. 5/2007. P. 55.

[6] Tridukh G.M., Prisakar A.M., Andriesh A.M., Nastas A.M. Inventor's certificate MD № 341 Z, BOPI nr. 2/2011.

[7] Nastas A., Andries A., Bivol V., Prisacari A., Triduh G. // J. Optoelectronics and Advanced Materials. 2005. V. 7. N 4. P. 1887.

[8] Настас А.М., Андриеш А.М., Бивол В.В., Присакар А.М., Тридух Г.М. // ЖТФ. 2009. Т. 79. В. 2. C. 139; Nastas A.M, Andriesh A.M., Bivol V.V., Prisakar A.M., Tridukh G.M. // Tech. Phys. 2009. V. 54. N 2. P. 305.

[9] Настас А.М., Иову М.С., Тридух Г.М., Присакар А.М. // ЖТФ. 2015. Т. 85. В. 3. С. 148; Nastas A.M., Iovu M.S., Tridukh G.M., Prisakar A.M. // Technical Physics. 2015. V. 60. N 3. P. 466.

[10] Настас А.М., Иову М.С., Присакар А.М. // Письма в ЖТФ. 2014. Т. 40. В. 9. С. 80; Nastas A.M., Iovu M.S., Prisakar A.M. // Technical Physics Lett. 2014. V. 40. N 5. P. 401.
[11] Настас А.М., Йову М.С., Присакар А.М., Тридух Г.М. // ЖТФ. 2017. Т. 87. В. 9. С. 1395-1398; Nastas A.M., Iovu M.S., Prisakar A.M., Tridukh G.M. // Technical Physics. 2017. V. 62. N 9. P. 1403.

[12] Bodurov I., Yovcheva T., Vlaeva I., Viraneva A., Todorov R., Spassov G., Sainov S. // J. Physics: Conference Ser. 2012. V. 398. art. N 012053, 6 pp.

[13] Stetsun A.I., Dvorina L.A. // Semiconductors. 2011. V. 45. N 10. P. 1291.

[14] Lyubin V., Arsh A., Klebanov M., Sroumin N., Kantarovich K., Bar I., Dror R., Sfez B. // J. Optoelectronics and Advanced Materials. 2008. V. 10. N 12. P. 3182. 